\title{
Pacific
}

Journal of

Mathematics

\section{HOMOGENEOUS RICCI POSITIVE 5-MANIFOLDS}

Dimitri Alekseevsky, Isabel Dotti de Miatello

AND CARLOS J. FERRARIS 


\title{
HOMOGENEOUS RICCI POSITIVE 5-MANIFOLDS
}

\author{
D. Alekseevsky, Isabel Dotti and C. Ferraris
}

We classify all 5-dimensional homogeneous Riemannian manifolds with positive Ricci curvature and among these we determine all Einstein manifolds. A new Einstein metric is found.

\section{Introduction.}

It is well known that a 2 or 3 dimensional Einstein manifold is of constant curvature. On the other hand it was proved by Jensen $[\mathbf{J} 1]$ that a 4-dimensional homogeneous Einstein manifold is symmetric. In dimension 5, Wang and Ziller showed that $S^{2} \times S^{3}$ admits infinitely many $S^{3} \times S^{3}$ invariant and non-isometric Einstein metrics with positive scalar curvature ([W-Z]). The purpose of this paper is to classify the 5-dimensional homogeneous Riemannian manifolds with positive Ricci curvature and to determine those which are Einstein (see [B], 7.4.2 and following statements). We will first show that this problem reduces to studying the $S^{3} \times S^{3}$-invariant metrics on $S^{3} \times S^{3} / S^{1}$, by analyzing the various possibilities (see $\S 3$ ).

The $S^{3} \times S^{3}$ invariant metrics in $S^{3} \times S^{3} / S^{1}$ split naturally into two types, diagonal and non diagonal. For every imbedding of $S^{1}$ into $S^{3} \times S^{3}$ there exists a unique Einstein metric of diagonal type. They coincide (see Remark 2) with those considered in [W-Z]. When the isotropy representation is multiplicity free only the diagonal type occurs (see Proposition 4.1). This family was also considered by E.Rodionov in $[\mathbf{R}]$. When the isotropy representation has equivalent subrepresentations both types of metrics (diagonal and non-diagonal) occur. We find that, in the class of non-diagonal metrics there is a new (unique up to homotethy) Einstein metric.

\section{The self-adjoint Ricci transformation.}

If $M$ is a connected manifold, a Riemannian metric on $M$ is called homogeneous if the isometry group $I(M)$ acts transitively.

Let $M$ be a manifold with a homogeneous metric of positive Ricci curvature. Then, the theorem of Bonnet-Myers implies that $M$ is compact. Moreover, there exist a compact, connected and semisimple Lie subgroup of $I(M)$ acting transitively and effectively by isometries on $M([\mathbf{D M}])$. Thus, 
$M$ is diffeomorphic to $G / H$ where $G$ is a compact, connected and semisimple Lie group, $H$ is the isotropy subgroup at a point in $M$ and the action of $G$ on $G / H$ is effective. The Riemannian metric on $M$ gives rise to a $G$-invariant metric on $G / H$, that is, a Riemannian metric such that left translations by elements of $G$ are isometries.

Given a homogeneous space $G / H$ as above one can describe all its $G$ invariant metrics as follows. Let $\mathcal{G}, h$ be the Lie algebras of $G$ and $H$ and let $\mathcal{G}=h \oplus m$ be an $A d(H)$-invariant splitting of $\mathcal{G}$. If $\pi: G \rightarrow G / H$ denotes the quotient map, then $\pi_{*}$ identify $m$ with the tangent space of $G / H$ at the identity coset $\mathrm{eH}$ and the linear isotropy representation, which assigns to each $h \in H$ its differential at $e H$, corresponds to the adjoint representation of $H$ on $m$.

Thus, every $G$-invariant metric on $G / H$ corresponds to an $A d(H)$-invariant inner product on $m$ and conversely, every such product can be translated to all of $G / H$ to produce a $G$-invariant metric.

To compute the self-adjoint Ricci transformation of a $G$-invariant metric on $G / H$ we consider the corresponding $A d(H)$-invariant inner product on $m$ (hence $\pi_{*}$ is orthogonal) and extend this inner product to $\mathcal{G}$ by requiring it to be $\operatorname{Ad}(H)$-invariant and so that the decomposition $\mathcal{G}=h \oplus m$ be orthogonal. If $G$ is endowed with the left invariant metric obtained by translating the inner product defined on $\mathcal{G}$, then $\pi: G \rightarrow G / H$ is a Riemannian submersion with totally geodesic fibers. We may thus apply Proposition 1 of [DM] and obtain the following expression for the self-adjoint Ricci transformation $\hat{r}$ in $T_{e H}(G / H)$,

$$
\begin{aligned}
\left\langle\hat{r} \pi_{*} x, \pi_{*} y\right\rangle= & -\frac{1}{2} \operatorname{tr} a d_{x} a d_{y}-\frac{1}{2} \sum_{i}\left\langle\left[x, h_{i}\right]_{m},\left[y, h_{i}\right]_{m}\right\rangle \\
& +\frac{1}{2} \sum_{i<j}\left\langle\left[h_{i}, h_{j}\right], x\right\rangle\left\langle\left[h_{i}, h_{j}\right], y\right\rangle
\end{aligned}
$$

where $\left\{h_{i}\right\}$ is an orthonormal basis of $m$ and $x, y$ are vectors in $m$.

One can verify easily using (I) that normal metrics (i.e. induced by biinvariant ones) have positive Ricci curvature in any quotient of a compact semisimple Lie group.

\section{Homogeneous manifolds of positive Ricci curvature.}

It follows from $\S 2$ that in order to find all manifolds admitting a homogeneous metric of positive Ricci curvature we need to determine, up to diffeomorphism, all homogeneous spaces $G / H$ where $G$ is a compact, connected and semisimple Lie group and $H$ is a closed subgroup of $G$ containing no 
non trivial invariant subgroups of $G$.

Since having positive Ricci curvature is a local property we will assume that $G / H$ is simply connected; this in turn implies that $H$ is connected. Therefore, the imbedding of $H$ in $G$ will be determined by that of $h$ in $\mathcal{G}$.

If $G / H$ is 5-dimensional, the isotropy representation is a one to one homomorphism $A d: H \rightarrow S O(5)$ with derivative denoted by $J: h \rightarrow s o(5)$. Since $h$ is the Lie algebra of a compact Lie group, it decomposes $h=z \oplus \bar{h}, z$ the center of $h$ and $\bar{h}$ semisimple. We recall that the semisimple real Lie algebras of dimension $\leq 10$ are $s u(2)$, so (4), so(4) $\oplus s u(2)$, su (3) and $s o(5)$. Now, the rank of $s o(5)$ is 2 hence the rank of $z \oplus \bar{h} \leq 2$. In particular, $\operatorname{dim} z \leq 2$ and $\operatorname{rank} \bar{h} \leq 2$. Thus, the possibilities for $h$ we need to consider are
(1) $h=\mathbf{R}$
(2) $h=\mathbf{R}^{2}$
(3) $h=s u(2)$
(4) $h=\mathbf{R} \oplus s u(2)$
(5) $h=s u(2) \oplus s u(2)$
(6) $h=s u(3)$
(7) $h=s o(5)$.

We now analyze the different cases.

(1) If $h=\mathbf{R}$ then $\mathcal{G}=s u(2) \oplus s u(2)$. It is well known that all closed connected subgroups $H$ in $S^{3} \times S^{3}$ with $h=\mathbf{R}$ are given by

$$
H=\alpha_{\left(m, m^{\prime}\right)} S^{1}=\left\{\left(z^{m}, z^{m^{\prime}}\right), z \in S^{1}\right\}
$$

where either $m=1, m^{\prime}=0$, or $m=0, m^{\prime}=1$, or $\operatorname{gcd}\left(m, m^{\prime}\right)=1$. Thus, in this situation, the homogeneous spaces $M_{\left(m, m^{\prime}\right)}=S^{3} \times S^{3} /_{\alpha_{\left(m, m^{\prime}\right)}} S^{1}$ we obtain are all diffeomorphic to $S^{3} \times S^{2}$ (see [Sm]). We will study all $S^{3} \times S^{3}$-invariant metrics on $M_{\left(m, m^{\prime}\right)}$ in $\S 4$.

(2) Can not hold since if $h=\mathbf{R}^{2}$ then $\operatorname{dim} \mathcal{G}=7$ and there are no 7dimensional semisimple Lie algebras.

(3) If $h=s u(2)$ then $\mathcal{G}=s u(3)$. The restriction of the tautological representation of $s u(3)$ to $h$ gives a complex representation of $h=s u(2)$ in $\mathbf{C}^{3}$. There are two such representations: a) reducible with decomposition $\mathbf{C}^{3}=\mathbf{C}^{2} \oplus \mathbf{C}^{1}$ and b) irreducible, equivalent to the symmetric square of the tautological representation of $s u(2)$. Thus, all connected $H$ in $S U$ (3) with $h=s u(2)$ are given by

$$
\left\{\left(\begin{array}{ll}
1 & 0 \\
0 & A
\end{array}\right): A \in S U(2)\right\},\left\{\left(\begin{array}{cc}
A & 0 \\
0 & A
\end{array}\right): A \in S O(3)\right\} .
$$

(4) Let $h=\mathbf{R} \oplus s u(2)$. Then $\mathcal{G}=s u(2) \oplus s u(2) \oplus s u(2)$. Denote by $\alpha: h \rightarrow \mathcal{G}$ a one to one Lie algebra homomorphism and by $p_{i}, i=1,2,3$, the projection of $\mathcal{G}$ onto its i-th component. Then, up to permutation, there are only three possibilities for the images $p_{i} \alpha(s u(2)), \quad i=1,2,3$

1. $(s u(2), s u(2), s u(2))$, 
2. $(s u(2), s u(2), 0)$,

3. $(s u(2), 0,0)$.

The first case is impossible since the centralizer of $\alpha(s u(2))$ would be trivial. The third case can not occur, otherwise $\alpha(s u(2))$ would be an ideal of $\mathcal{G}$. Thus, only the second case remains and one obtains that, the 5-dimensional, simply connected homogeneous Riemannian manifold $G / H$ with $h=\mathbf{R} \oplus s u(2)$ is the direct product $S^{3} \times S^{3} / \Delta S^{3} \times S^{3} / S^{1}$, of standard spheres. (Here $\Delta S^{3}$ denotes the diagonal subgroup of the group $S^{3} \times S^{3}$.)

(5) If $h=s u(2) \oplus s u(2), \mathcal{G}$ has to be $s u(2) \oplus s u(3)$ and one can construct ideals of $\mathcal{G}$ contained in a copy of $h$, contradicting effectiveness. To prove this last assertion let $\alpha: h \rightarrow \mathcal{G}$ be a one to one homomorphism, let $p_{1}: \mathcal{G} \rightarrow$ $s u(2)$ and $p_{2}: \mathcal{G} \rightarrow s u(3)$ denote the projections and consider the ideals $h_{1}=\operatorname{ker} p_{1} \alpha$ and $h_{2}=\operatorname{ker} p_{2} \alpha$. Furthermore, it is known (see [G-G]) that $s u(3)$ does not have $s u(2) \oplus s u(2)$ as a subalgebra. Hence $h_{1}$ is an ideal of $h$ isomorphic to $s u(2)$. We may also assume that $h_{1}=\{0\} \oplus s u(2)$. Then the centralizer of $h_{1}$ in $\mathcal{G}$ is given by $s u(2) \oplus \mathbf{R}$. In particular the centralizer of $h_{1}$ in $h, s u(2) \oplus\{0\}$, is an ideal of $\mathcal{G}$, contradicting effectiveness. Thus (5) is impossible.

(6) Can not hold since every two dimensional representation of $s u(3)$ is trivial and $s u(3)$ has rank 2.

(7) If $h=s o(5)$ then the isometry group has dimension $\geq 15$ and therefore $G / H$ is the sphere of constant curvature, $S O(6) / S O(5)($ see $[\mathbf{K}-\mathbf{N}]$, p. 308).

We are now in a position to list the simply connected 5-dimensional manifolds admitting homogeneous metrics of positive Ricci curvature.

$G \quad H \quad M$

$\begin{array}{llll}(\mathrm{I}) & S U(2) \times S U(2) & \alpha_{\left(m, m^{\prime}\right)} S^{1} & S^{3} \times S^{2} \\ (\mathrm{II}) & S U(3) & S U(2) & S^{5} \\ (\mathrm{III}) & S U(3) & S O(3) & S U(3) / S O(3) \\ (\mathrm{IV}) & S U(2) \times S U(2) \times S U(2) & \Delta S U(2) \times S O(2) & S^{3} \times S^{2} \\ (\mathrm{~V}) & S O(6) & S O(5) & S^{5} .\end{array}$

The corresponding homogeneous metrics in case (I) are obtained in $\S 4$, Lemma 4.1 and the associated selfadjoint Ricci transformation in Proposition 4.1. In cases II through $\mathrm{V}$ the inclusions are the standard ones. In case II the isotropy representation is a 1-dimensional trivial plus an irreducible one, and the metrics have been studied by Jensen in [J2]. He showed that the only Einstein metric is that of constant curvature. We note that this one 
is not induced by the Killing form in G. Finally, the cases III, IV, and V correspond to symmetric spaces.

\section{Ricci curvatures of $S^{3} \times S^{3}$-invariant metrics.}

We recall from $\S 3$ that $M_{\left(m, m^{\prime}\right)}$ denotes the space $S^{3} \times S^{3} /_{\alpha_{\left(m, m^{\prime}\right)}} S^{1}$ where $\alpha_{\left(m, m^{\prime}\right)}(z)=\left(z^{m}, z^{m^{\prime}}\right)$ and either $m=0, m^{\prime}=1$ or $\operatorname{gcd}\left(m, m^{\prime}\right)=1$. When $m=0, m^{\prime}=1$ (or $m=1, m^{\prime}=0$ ), the $S^{3} \times S^{3}$-invariant metrics on $M_{(0,1)}=S^{3} \times S^{3} / S^{1}$ are given by $\left(g_{1}, g_{2}\right)$ where $g_{1}$ is a left invariant metric on $S^{3}$ (see [M]) and $g_{2}$ is the standard metric on $S^{2}$. In order to find all $S^{3} \times S^{3}$-invariant metrics on $M_{\left(m, m^{\prime}\right)}, \operatorname{gcd}\left(m, m^{\prime}\right)=1$ and their Ricci curvatures, we start by fixing some notation.

Let $\mathcal{G}=s u(2) \oplus s u(2)$ be the Lie algebra of $G=S^{3} \times S^{3}$ equipped with the bi-invariant metric $(x, y)=-\frac{1}{2} B(x, y)$ where $B$ is the Killing form and let $\underline{t}_{\left(m, m^{\prime}\right)}$ be the Lie algebra of $T_{\left(m, m^{\prime}\right)}=\alpha_{\left(m, m^{\prime}\right)} S^{1}$. We denote by $\left\{t, e_{+}, e_{-}\right\}$ (respectively $\left\{t^{\prime}, e_{+}^{\prime}, e_{-}^{\prime}\right\}$ ) an orthonormal basis of the first $s u(2)$ factor in $\mathcal{G}$ (respectively, the second $s u(2)$ factor in $\mathcal{G}$ ) satisfying the relations

$$
\left[t, e_{+}\right]=e_{-},\left[e_{+}, e_{-}\right]=t,\left[e_{-}, t\right]=e_{+}
$$

(respectively $\left.\left[t^{\prime}, e_{+}^{\prime}\right]=e_{-}^{\prime},\left[e_{+}^{\prime}, e_{-}^{\prime}\right]=t^{\prime},\left[e_{-}^{\prime}, t^{\prime}\right]=e_{+}^{\prime}\right)$.

Set

$v_{\left(m, m^{\prime}\right)}=m t+m^{\prime} t^{\prime}, \underline{m}=\operatorname{span}\left\{t, e_{+}, e_{+}^{\prime}, e_{-}, e_{-}^{\prime}\right\}, J_{\left(m, m^{\prime}\right)}=a d_{v_{\left(m, m^{\prime}\right)}} \underline{\mid m}$.

Then it is clear that $t_{\left(m, m^{\prime}\right)}=\mathbf{R} v_{\left(m, m^{\prime}\right)}$ and $\underline{m}$ is an $A d\left(T_{\left(m, m^{\prime}\right)}\right)$-invariant complement. Moreover the matrix of $J_{\left(m, m^{\prime}\right)}$ with respect to the basis $\left\{t, e_{+}, e_{+}^{\prime}, e_{-}, e_{-}^{\prime}\right\}$ is

$$
\left[J_{\left(m, m^{\prime}\right)}\right]=\left[\begin{array}{c|cccc}
0 & \multicolumn{5}{|c}{0} \\
\hline & 0 & 0 & -m & 0 \\
0 & 0 & 0 & 0 & -m^{\prime} \\
& m & 0 & 0 & 0 \\
& 0 & m^{\prime} & 0 & 0
\end{array}\right]
$$

Convention: When $m=m^{\prime}=1$ we set $v=v_{(1,1)}, \underline{t}=\underline{t}_{(1,1)}$ and $J=J_{(1,1)}$.

We note that $\bar{J}$ defined on $\mathbf{R} s \times \underline{m}$ by $\bar{J} s=t, \bar{J} t=-s$ and $\bar{J}=J$ on the orthogonal complement (with respect to the Killing form) of $\mathbf{R} t$ in $\underline{m}$ give rise to an invariant complex structure on $\mathbf{R} \times M_{\left(m, m^{\prime}\right)}$. 
Let $A_{r, s, a, b}$ denote the real matrix

$$
A_{r, s, a, b}=\left[\begin{array}{cc|cc}
\mathrm{r} & \mathrm{a} & 0 & \mathrm{~b} \\
\mathrm{a} & \mathrm{s} & -\mathrm{b} & 0 \\
\hline 0 & -\mathrm{b} & \mathrm{r} & \mathrm{a} \\
\mathrm{b} & 0 & \mathrm{a} & \mathrm{s}
\end{array}\right] .
$$

Lemma 4.1. The set of all $S^{3} \times S^{3}$-invariant metrics on $M_{\left(m, m^{\prime}\right)}$, $\operatorname{gcd}\left(m, m^{\prime}\right)=1$, when expressed as symmetric matrices with respect to the basis $\left\{t, e_{+}, e_{+}^{\prime}, e_{-}, e_{-}^{\prime}\right\}$ are given by

$$
B_{\varepsilon, r, s, a, b}=\left[\begin{array}{c|c}
\varepsilon & \\
\hline & A_{r, s, a, b}
\end{array}\right]
$$

with $\varepsilon>0, r>0, r s>a^{2}+b^{2}$ and $a^{2}+b^{2}=0$ if $m \neq m^{\prime}$. When $m=$ $m^{\prime}=1$, the inner automorphism $I_{w}, w=\left(e^{i \theta / 4}, e^{-i \theta / 4}\right)$ induces an isometry between the metrics corresponding to $B_{\varepsilon, r, s, \rho, 0}$ and $B_{\varepsilon, r, s, \operatorname{Re} \rho e^{i}} \theta_{, \operatorname{Im} \rho e^{-i}} \theta \cdot$

The proof is straightforward and will be omitted.

\section{Notes:}

1. The metrics for which $a^{2}+b^{2}=0$ (respectively, $a^{2}+b^{2} \neq 0$ ) in Lemma 4.1 were referred to as being of diagonal type (respectively, of non diagonal type) in the introduction.

$\dot{2}$. The manifolds $M_{\left(m, m^{\prime}\right)}$ appear in the classification of 5-dimensional $\phi$-symmetric spaces $[\mathbf{K}-\mathbf{W}]$, as admitting invariant Sasakian metrics. It can be shown that for some choices of $\varepsilon, r, s$ the matrix $B_{\varepsilon, r, s, 0,0}$ induces a Sasakian metric.

Given a matrix $B_{\varepsilon, r, s, a, b}$ as in Lemma 4.1 , and if $\lambda, \mu, \lambda \geq \mu$, are the roots of $p_{r, s, a, b}(t)=\operatorname{det}\left(A_{r, s, a, b}-t I\right)$ then

$$
(r-t)(s-t)-\left(a^{2}+b^{2}\right)=(t-\lambda)(t-\mu)
$$

from which it immediately follows that

$$
\begin{aligned}
r+s= & \lambda+\mu, \quad \lambda \mu=r s-\left(a^{2}+b^{2}\right), \\
a^{2}+b^{2} & =(\lambda-s)(\lambda-r)=(r-\mu)(s-\mu) \\
& =(\lambda-r)(r-\mu)=(\lambda-s)(s-\mu) \\
\lambda \neq \mu \quad & \text { when } a^{2}+b^{2} \neq 0 .
\end{aligned}
$$


If $a^{2}+b^{2}=0$, then $\left\{t, e_{+}, e_{+}^{\prime}, e_{-}, e_{-}^{\prime}\right\}$ is an orthogonal basis of eigenvectors. When $a^{2}+b^{2} \neq 0$ (hence $m=m^{\prime}=1$ ), an orthogonal basis of eigenvectors for $B_{\varepsilon, r, s, a, b}$ is given by $\left\{t, f_{+}, f_{+}^{\prime}, f_{-}, f_{-}^{\prime}\right\}$, where

$$
\begin{aligned}
& f_{+}=-a e_{+}+(r-\lambda) e_{+}^{\prime}+b e_{-}, f_{-}=J f_{+} \\
& f_{+}^{\prime}=-a e_{+}+(r-\mu) e_{+}^{\prime}+b e_{-}, f_{-}^{\prime}=J f_{+}^{\prime} .
\end{aligned}
$$

The following bracket relations will be useful to compute Ricci curvatures in Proposition 4.1. They can be obtained directly using (II) and (IV).

$$
\begin{aligned}
{\left[f_{+}, f_{+}^{\prime}\right] } & =\left[f_{-}, f_{-}^{\prime}\right]=0, \\
{\left[t, f_{+}\right] } & =\left[t, f_{+}^{\prime}\right]=\frac{1}{(\lambda-\mu)}\left[(r-\mu) f_{-}+(\lambda-r) f_{-}^{\prime}\right], \\
{\left[t, f_{-}\right] } & =\left[t, f_{-}^{\prime}\right]=J\left[t, f_{+}\right], \\
{\left[f_{+}, f_{-}\right]_{\underline{m}} } & =(\lambda-r)(r-s) t, \\
{\left[f_{+}^{\prime}, f_{-}^{\prime}\right]_{\underline{m}} } & =(r-\mu)(s-r) t, \\
{\left[f_{+}, f_{-}^{\prime}\right]_{\underline{m}} } & =-\left[f_{-}, f_{+}^{\prime}\right]_{\underline{m}}=2\left(a^{2}+b^{2}\right) t .
\end{aligned}
$$

If one sets

$$
\begin{aligned}
& m_{1}=\operatorname{span}\left\{e_{+}, e_{+}^{\prime}\right\} \quad m_{2}=\operatorname{span}\left\{e_{-}, e_{-}^{\prime}\right\} \\
& p_{1}=\operatorname{span}\left\{f_{+}, f_{+}^{\prime}\right\} p_{2}=\operatorname{span}\left\{f_{-}, f_{-}^{\prime}\right\}
\end{aligned}
$$

then the decompositions

$$
m=\mathbf{R} t \oplus m_{1} \oplus m_{2}=\mathbf{R} t \oplus p_{1} \oplus p_{2}
$$

are orthogonal with respect to the Killing form $B$ and it follows from (II) and (V) that they satisfy

$$
\begin{aligned}
{\left[m_{i}, m_{i}\right] } & =\left[p_{i}, p_{i}\right]=0, i=1,2 \\
{\left[\mathbf{R} t, m_{1}\right] } & =\mathbf{R} e_{-}, \quad\left[\mathbf{R} t, m_{2}\right]=\mathbf{R} e_{+} \\
{\left[\mathbf{R} t, p_{1}\right]_{m} } & =p_{2}, \quad\left[\mathbf{R} t, p_{2}\right]_{m}=p_{1} \\
{\left[m_{1}, m_{2}\right]_{m} } & =\mathbf{R} t=\left[p_{1}, p_{2}\right]_{m} .
\end{aligned}
$$

Let $\langle$,$\rangle be the inner product on m$ given by $\langle x, y\rangle=\left(x, B_{\varepsilon, r, s, a, b} y\right)$. Then it is clear, by the choice of the subspaces $m_{i}, p_{i}, i=1,2$ that $m=$ $\mathbf{R} t \oplus m_{1} \oplus m_{2}$ (resp. $m=\mathbf{R} t \oplus p_{1} \oplus p_{2}$ ) is an orthogonal decomposition with respect to $\langle$, $\rangle$ when $a^{2}+b^{2}=0$ (resp. when $a^{2}+b^{2} \neq 0$ ). Moreover $m_{2}=J_{\left(m, m^{\prime}\right)} m_{1}$ and $p_{2}=J p_{1}$. 
Lemma 4.2. The Ricci transformation $\hat{r}$, associated to $\langle x, y\rangle=$ $\left(x, B_{\varepsilon, r, s, a, b}(y)\right)$ verifies

(i) $t$ is an eigenvector of $\hat{r}$,

(ii) for any choice of $\varepsilon, r, s, \hat{r}$ preserves the decomposition $m_{1} \oplus m_{2}$ (resp. $p_{1} \oplus p_{2}$ ) when $a^{2}+b^{2}=0$ (resp. $a^{2}+b^{2} \neq 0$ ) and the eigenvalues of $\hat{r}$ in $m_{1}$ (resp. $p_{1}$ ) coincide with the eigenvalues of $\hat{r}$ in $m_{2}$ (resp. $\left.p_{2}\right)$ when $a^{2}+b^{2}=0\left(\right.$ resp. $\left.a^{2}+b^{2} \neq 0\right)$.

Proof. Since $\hat{r}$ commutes with $J_{\left(m, m^{\prime}\right)}, \operatorname{gcd}\left(m, m^{\prime}\right)=1$, (i) follows. To show that $\hat{r}$ preserves the decomposition $m_{1} \oplus m_{2}$ (resp. $p_{1} \oplus p_{2}$ ) we apply (I) to a pair of vectors $x \in m_{1}$ (resp. $p_{1}$ ) $y \in m_{2}$ (resp. $p_{2}$ ) and then use (VI) together with the fact that the decomposition $\mathbf{R} t \oplus m_{1} \oplus m_{2}$ (resp. $\left.\mathbf{R} t \oplus p_{1} \oplus p_{2}\right)$ is orthogonal with respect to both metrics $($,$) and \langle$,$\rangle when$ $a^{2}+b^{2}=0$ (resp. $a^{2}+b^{2} \neq 0$ ). The last assertion in (ii) follows since $\hat{r}$ commutes with $J_{\left(m, m^{\prime}\right)}$ and $m_{2}=J_{\left(m, m^{\prime}\right)} m_{1}, p_{2}=J p_{1}$.

The previous lemma together with the next proposition provide the Ricci curvatures of any $S^{3} \times S^{3}$-invariant metric on $M_{\left(m, m^{\prime}\right)}$.

Set $p=e\left(\alpha_{\left(m, m^{\prime}\right)} S^{1}\right)$.

Proposition 4.1. The self-adjoint Ricci transformation $\hat{r}$ on $T_{p} M_{\left(m, m^{\prime}\right)}$ associated to the metric given by $B_{\varepsilon, r, s, a, b}$ with respect to the orthogonal basis $\left\{t, e_{+}, e_{+}^{\prime}, e_{-}, e_{-}^{\prime}\right\}$ if $a^{2}+b^{2}=0$ is given by $\hat{r}=\operatorname{diag}\left(r_{0}, r_{1}, r_{2}, r_{1}, r_{2}\right)$ where

$$
\begin{aligned}
& r_{0}=\langle\hat{r} t, t\rangle=\frac{\varepsilon^{2}}{2}\left(\frac{1}{r^{2}}+\frac{1}{s^{2}}\left(\frac{m}{m^{\prime}}\right)^{2}\right), \\
& r_{1}=\left\langle\hat{r} e_{+}, e_{+}\right\rangle=\frac{1}{2}\left(2-\frac{\varepsilon}{r}\right), \\
& r_{2}=\left\langle\hat{r} e_{+}^{\prime}, e_{+}^{\prime}\right\rangle=\frac{1}{2}\left(2-\left(\frac{m}{m^{\prime}}\right)^{2} \frac{\varepsilon}{s}\right) .
\end{aligned}
$$

If $a^{2}+b^{2} \neq 0$ (hence $m=m^{\prime}$ ), with respect to the orthogonal basis $\left\{t, f_{+}, f_{+}^{\prime}, f_{-}, f_{-}^{\prime}\right\}, \hat{r}=\operatorname{diag}\left(r_{0}, R, R\right), R=\left(r_{i j}\right)$ a two by two symmetric matrix where, if $\lambda \geq \mu$ are the roots of $p_{r, s, a, b}(t)$ (see (III))

$$
\begin{aligned}
r_{0} & =\langle\hat{r} t, t\rangle=\frac{1}{\lambda \mu}\left\{-\left(a^{2}+b^{2}\right)+\frac{\varepsilon^{2}}{2 \lambda \mu}\left[(\lambda-\mu)^{2}+2 \lambda \mu-4\left(a^{2}+b^{2}\right)\right]\right\} \\
r_{1,2} & =\left\langle\hat{r} f_{+}, f_{+}^{\prime}\right\rangle=\frac{\left(a^{2}+b^{2}\right)(s-r)\left(\lambda \mu-2 \varepsilon^{2}\right)}{2 \varepsilon \lambda \mu} \\
r_{1,1} & =\frac{1}{\left\|f_{+}\right\|^{2}}\left\langle\hat{r} f_{+}, f_{+}\right\rangle=\frac{1}{\lambda}-\frac{\varepsilon}{2 \lambda^{2}}+\left(a^{2}+b^{2}\right) \frac{\left[(\lambda+\mu) \lambda-4 \varepsilon^{2}\right]}{2(\lambda-\mu) \mu \varepsilon \lambda^{2}}
\end{aligned}
$$




$$
r_{2,2}=\frac{1}{\left\|f_{+}^{\prime}\right\|^{2}}\left\langle\hat{r} f_{+}^{\prime}, f_{+}^{\prime}\right\rangle=\frac{1}{\mu}-\frac{\varepsilon}{2 \mu^{2}}+\left(a^{2}+b^{2}\right) \frac{\left[(\lambda+\mu) \mu-4 \varepsilon^{2}\right]}{2(\mu-\lambda) \lambda \varepsilon \mu^{2}} .
$$

The proof consists of a tedious and lengthly but straightforward calculation and we will omit it.

As a corollary we obtain

Corollary 4.1. The metric $B_{\varepsilon, r, s, a, b}$ has positive Ricci curvature if and only if $2 r>\varepsilon, 2\left(\frac{m^{\prime}}{m}\right)^{2} s>\varepsilon$ when $a^{2}+b^{2}=0$ or $r_{0}>0, r_{1,1}>0, \operatorname{det} R>0$ when $a^{2}+b^{2} \neq 0$.

Theorem 4.1. (i) $M_{\left(m, m^{\prime}\right)}, \operatorname{gcd}_{\left(m, m^{\prime}\right)}=1, m \neq m^{\prime}$ admits a unique $S^{3} \times S^{3}$-invariant Einstein metric.

(ii) $M_{(1,1)}$ admits two $S^{3} \times S^{3}$-invariant Einstein metrics. (Compare [B] page 472.)

Proof. Because of Proposition 4.1, the analysis of the Einstein condition will be considered separately in two cases: $a^{2}+b^{2}=0$ and $a^{2}+b^{2} \neq 0$.

Case 1. Since in this case $\hat{r}$ is diagonal, the Einstein condition becomes

$$
\frac{\varepsilon}{2}\left(\frac{1}{r^{2}}+\frac{1}{s^{2}} c^{2}\right)=\frac{1}{2 r}\left(2-\frac{\varepsilon}{r}\right)=\frac{1}{2 s}\left(2-c^{2} \frac{\varepsilon}{s}\right)
$$

where $c=\frac{m}{m^{\prime}}$. Then one easily checks that solving (VII) is equivalent to finding $(s, r, \varepsilon)$ satisfying

$$
\begin{aligned}
& f_{1}(s, r, \varepsilon)=s^{2}(2 r-\varepsilon)-s\left(2 r^{2}\right)+r^{2} c^{2} \varepsilon=0 \\
& f_{2}(s, r, \varepsilon)=s^{2}(2 r-2 \varepsilon)-r^{2} c^{2} \varepsilon=0 .
\end{aligned}
$$

Set $g_{1}(s, r, \varepsilon)=\left(f_{1}+f_{2}\right)(s, r, \varepsilon)$ and $g_{2}(s, r, \varepsilon)=\left(f_{1}-f_{2}\right)(s, r, \varepsilon)$. Since $g_{1}(s, r, \varepsilon)=s\left[s(4 r-3 \varepsilon)-2 r^{2}\right]$ it follows that $s=2 r^{2} / 4 r-3 \varepsilon$ with $r>$ $3 \varepsilon / 4$ is a solution to $g_{1}(s, r, \varepsilon)=0$. On the other hand the discriminant of $g_{2}(s, r, \varepsilon)=s^{2} \varepsilon-s\left(2 r^{2}\right)+2 r^{2} c^{2} \varepsilon$ is $\Delta=4 r^{2}\left(r^{2}-2 \varepsilon^{2} c^{2}\right)$, hence $r$ and $\varepsilon$ must also verify $r^{2}>2 \varepsilon^{2} c^{2}$. If we substitute $s=2 r^{2} / 4 r-3 \varepsilon$ into $g_{2}(s, r, \varepsilon)$ we obtain

$$
h(r, \varepsilon)=\frac{16 r^{2}}{(4 r-3 \varepsilon)^{2}}\left[-r^{3}+r^{2} \varepsilon\left(1+2 c^{2}\right)-r 3 c^{2} \varepsilon^{2}+\frac{9}{8} c^{2} \varepsilon^{3}\right]
$$




$$
=\frac{16 r^{2}}{(4 r-3 \varepsilon)^{2}} h_{1}(r, \varepsilon)
$$

Evaluating $h_{1}\left(\frac{3}{4} \varepsilon, \varepsilon\right)$ and $h_{1}(\sqrt{2}|c| \varepsilon, \varepsilon)$ we obtain

$$
h_{1}\left(\frac{3}{4} \varepsilon, \varepsilon\right)=\frac{9}{16.4} \varepsilon^{3}>0, \quad h_{1}(\sqrt{2}|c| \varepsilon, \varepsilon)=4 c^{2} \varepsilon^{3}\left(|c|-\frac{5}{8} \sqrt{2}\right)^{2}>0
$$

if $\varepsilon>0$ (note that $c=\frac{m}{m^{\prime}} \in \mathbf{Q}$ ). Since for any $\varepsilon>0, \lim _{r \rightarrow+\infty} h_{1}(r, \varepsilon)=-\infty$, there exists a root $r(\varepsilon)$ of the equation $h_{1}(r, \varepsilon)=0$ with $r(\varepsilon)>\frac{3}{4} \varepsilon$ and $r(\varepsilon)>\sqrt{2}|c| \varepsilon$. Thus, $\varepsilon, r(\varepsilon)$ and $s(\varepsilon)=2(r(\varepsilon))^{2} / 4 r(\varepsilon)-3 \varepsilon$ satisfy (VIII).

We show next that the root $r(\varepsilon)$ which was found above is unique.

Let $\varepsilon>0, \varepsilon$ fixed. The discriminant of $\frac{\partial}{\partial r} h_{1}(r, \varepsilon)=-3 r^{2}+2 r \varepsilon(1+$ $\left.2 c^{2}\right)-3 c^{2} \varepsilon^{2}$ is $\Delta=\left(1+2 c^{2}\right)^{2}-9 c^{2}$. Hence, if $|c|>1$ or $|c|>\frac{1}{2}, \Delta<0$ and $h_{1}$ is decreasing. When $\frac{1}{2}<|c|<1, h_{1}(r, \varepsilon)$ has two critical points, $r_{ \pm}=\frac{\varepsilon}{3}\left[\left(1+2 c^{2}\right) \pm \sqrt{\Delta}\right]$ and since $r_{-}<\sqrt{2} \varepsilon|c|, h_{1}$ has only one root greater than $\sqrt{2}|c| \varepsilon$. If $|c|=1, h_{1}(r, \varepsilon)=-(r-\varepsilon) 3+\left(\frac{\varepsilon}{2}\right)^{3}$ and if $|c|=\frac{1}{2}$, $h_{1}(r, \varepsilon)=-(r-\varepsilon)^{3}+\frac{5}{4}\left(\frac{\varepsilon}{2}\right)^{3}$ thus the claim is proved.

Finally, since $h_{1}(\varepsilon r(1), \varepsilon)=0$ one gets $\varepsilon r(1)=r(\varepsilon)$. Thus we obtain, when $m \neq m^{\prime}$, a unique $S^{3} \times S^{3}$-invariant Einstein metric on $M_{\left(m, m^{\prime}\right)}$. If $m=m^{\prime}, r(\varepsilon)=s(\varepsilon)=\frac{3}{2} \varepsilon$ and from (VII) it follows that the Einstein constant $E$ is $E=\frac{4}{9 \varepsilon}$.

Case 2. When $a^{2}+b^{2} \neq 0$, the Einstein condition becomes

$$
\begin{gathered}
\lambda \mu=2 \varepsilon^{2} \quad \text { or } \quad s=r \quad \text { and } \\
\frac{1}{\varepsilon \lambda \mu}\left\{-\left(a^{2}+b^{2}\right)+\frac{\varepsilon^{2}}{2 \lambda \mu}\left[(\lambda-\mu)^{2}+2 \lambda \mu-4\left(a^{2}+b^{2}\right)\right]\right\} \\
=\frac{1}{\lambda}-\frac{\varepsilon}{2 \lambda^{2}}+\left(a^{2}+b^{2}\right) \frac{\left[(\lambda+\mu) \lambda-4 \varepsilon^{2}\right]}{2(\lambda-\mu) \mu \varepsilon \lambda^{2}} \\
=\frac{1}{\mu}-\frac{\varepsilon}{2 \mu^{2}}+\left(a^{2}+b^{2}\right) \frac{\left[(\lambda+\mu) \mu-4 \varepsilon^{2}\right]}{2(\mu-\lambda) \lambda \varepsilon \mu^{2}}
\end{gathered}
$$

If $\lambda \mu=2 \varepsilon^{2},(\mathrm{IX})$ is equivalent to

$$
\begin{aligned}
\frac{(\lambda-\mu)^{2}+4 \varepsilon^{2}-8\left(a^{2}+b^{2}\right)}{8 \varepsilon^{3}} & =\frac{1}{\lambda}-\frac{\varepsilon}{2 \lambda^{2}}+\frac{a^{2}+b^{2}}{4 \varepsilon^{3}} \\
& =\frac{1}{\mu}-\frac{\varepsilon}{2 \mu^{2}}+\frac{a^{2}+b^{2}}{4 \varepsilon^{3}} .
\end{aligned}
$$


One easily checks that $\frac{1}{\lambda}-\frac{\varepsilon}{2 \lambda^{2}}=\frac{1}{\mu}-\frac{\varepsilon}{2 \mu^{2}}$ if and only if $\lambda+\mu=4 \varepsilon$. Thus $\lambda=\varepsilon(2+\sqrt{2}), \mu=\varepsilon(2-\sqrt{2})$. Substituting the values for $\lambda$ and $\mu$ in the first expression in (X) we obtain $a^{2}+b^{2}=\varepsilon^{2}$. Now it is a routine to verify that $r=3 \varepsilon, \quad s=\varepsilon, a=\varepsilon \cos \theta, \quad b=\varepsilon \sin \theta$ gives a 1-parameter family of non homothetic Einstein metrics on $M_{(1,1)}$.

On the other hand, according to Lemma 4.1, they are all isometric to the metric given by $B_{\varepsilon, 3} \varepsilon, \varepsilon, \varepsilon, 0$ with Einstein constant $E$ equal to $\frac{1}{2 \varepsilon}$. In particular, on $M_{(1,1)}$, the Einstein metrics corresponding to $B_{\varepsilon, \frac{3}{2} \varepsilon, \frac{3}{2} \varepsilon, 0,0}$ and $B_{\varepsilon, 3 \varepsilon, \varepsilon, \varepsilon, 0}$ are not isometric.

To finish the proof we verify next that when $r=s$ there is no solution to the Einstein condition.

If $r=s$ then $\lambda=r+\left(a^{2}+b^{2}\right)^{1 / 2}, \quad \mu=r-\left(a^{2}+b^{2}\right)^{1 / 2}$. Set $\rho=$ $\left(a^{2}+b^{2}\right)^{1 / 2}$ and write $\lambda=r+\rho, \quad \mu=r-\rho, \quad \lambda+\mu=2 r, \lambda-\mu=$ $2 \rho, \lambda \mu=r^{2}-\rho^{2}$. (Note that $r^{2}>\rho^{2}$.) In this case (IX) is equivalent to

$$
\begin{aligned}
& \frac{1}{r+\rho}\left[1-\frac{\varepsilon}{2(r+\rho)}+\frac{\rho r}{2 \varepsilon(r-\rho)}-\frac{\rho \varepsilon}{r^{2}-\rho^{2}}\right]=\frac{\varepsilon^{2}-\rho^{2}}{\varepsilon\left(r^{2}-\rho^{2}\right)}= \\
& =\frac{1}{r-\rho}\left[1-\frac{\varepsilon}{2(r-\rho)}-\frac{\rho r}{2 \varepsilon(r+\rho)}+\frac{\rho \varepsilon}{r^{2}-\rho^{2}}\right] .
\end{aligned}
$$

From the first equality we obtain

$$
\frac{\varepsilon^{2}-\rho^{2}}{\varepsilon}=r-\rho-\frac{3}{2}+\frac{\rho r}{2 \varepsilon}
$$

and from the second one

$$
\frac{\varepsilon^{2}-\rho^{2}}{\varepsilon}=r+\rho-\frac{3}{2}-\frac{\rho r}{2 \varepsilon}
$$

Now the above two identities yield $r=2 \varepsilon$. But $\frac{\varepsilon^{2}-\rho^{2}}{\varepsilon}=2 \varepsilon+\rho-\frac{3}{2}-\rho=\frac{3}{2} \varepsilon$ gives $\rho^{2}<0$, thus a contradiction.

\section{Remarks.}

1. (i) of Theorem 4.1 was obtained by E. Rodionov in $[\mathbf{R}]$.

2. The metrics in $M_{\left(m, m^{\prime}\right)}$ which correspond to matrices with $a^{2}+b^{2}=0$ (see Lemma 4.1), when restricted to $m_{1} \oplus m_{2}$ are $A d\left(S^{1} \times S^{1}\right)$-invariant. Thus, they induce metrics in $S^{2} \times S^{2}$ of the form $r g \perp s g$, where $g$ is the standard metric in $S^{2}$. Wang and Ziller showed in [W-Z] that for some choices of $r, s$, the manifolds $M_{\left(m, m^{\prime}\right)}$ carry an Einstein metric uniquely characterized by the requirement that the projection onto $S^{2} \times S^{2}$ is a Riemannian submersion with totally geodesic fibers. Thus 
the Einstein metrics obtained in the case $a^{2}+b^{2}=0$ of Theorem 4.1 coincide with those previously obtained in [W-Z].

3. In [B], page 472, it is asserted that for every embedding of $S^{1}$ into $S^{3} \times S^{3}$ the quotient manifold $S^{3} \times S^{3} / S^{1}$ carries a unique $S^{3} \times S^{3}$ invariant Einstein metric. The statement above is true under the assumption that the isotropy representation has no equivalent subrepresentations. On the other hand (ii) of Theorem 4.1 shows that the diagonal embedding $z \rightarrow(z, z)$ of $S^{1}$ into $S^{3} \times S^{3}$ is exceptional.

\section{References}

[B] A. Besse, Einstein manifolds, Springer Verlag.

[DM] I. Dotti Miatello, Transitive group actions and Ricci curvature properties, Michigan Math. J., 35 (1988), 427-434.

[G-G] M. Goto and D. Grosshans, Semisimple Lie algebras, Marcel Dekker, New York, 1978.

[J1] G.R. Jensen, Homogeneous Einstein spaces of dimension 4, J. Diff. Geom., 3 (1971), 309-349.

[J2] —_ Einstein metrics on principal fibre bundles, J. Diff. Geom., 8 (1973), 599614.

[K-N] S. Kobayashi and K. Nomizu, Foundations of Differential Geometry, J.Wiley \& Sons, 1969.

[K-W] O. Kowalski and S. Wegrzynowski, A classification of five-dimensional $\phi$-symmetric spaces, Tensor, N.S, 46 (1987), 379-386.

[M] J. Milnor, Curvature properties of left invariant metrics, Advances in Math., 21 (1976), 293-329.

[R] E.D. Rodionov, Einstein metrics on a class of five dimensional homogeneous spaces, CMUC, Prague, 1991.

[Sm] S. Smale, On the strutures of 5-manifolds, Ann. of Math., 75 (1962), 38-46.

[W-Z] M. Wang and W. Ziller, Einstein metrics on principal torus bundles, J. Diff. Geom., 31 (1990), 215-248.

Received February 9, 1994 and revised June 29, 1995. The second author was partially supported by CONICET, CONICOR and ICTP.

Centre Sophus Lie

Moscow, Russia

AND

FAMAF, U.N.C.

Ciudad Universitaria

5000 Córdoba, Argentina

E-mail address: idotti@mate.uncor.edu

AND

UNIVERSIDADE DE FloRIANOPOLIS

Santa Catarina, Brazil 


\title{
PACIFIC JOURNAL OF MATHEMATICS
}

\author{
Founded in 1951 by
}

\author{
E. F. Beckenbach (1906-1982) $\quad$ F. Wolf (1904-1989)
}

\section{EDITORS}

Sun-Yung A. Chang (Managing Editor) Robert Finn University of California

Los Angeles, CA 90095-1555

pacific@math.ucla.edu

\section{F. Michael Christ}

University of California

Los Angeles, CA 90095-1555

christ@math.ucla.edu

Nicholas Ercolani

University of Arizona

Tucson, AZ 85721

ercolani@math.arizona.edu
Stanford University

Stanford, CA 94305

finn@gauss.stanford.edu

Steven Kerckhoff

Stanford University

Stanford, CA 94305

spk@gauss.stanford.edu

Martin Scharlemann

University of California

Santa Barbara, CA 93106

mgscharl@math.ucsb.edu

\section{Gang Tian}

Massachusettes Institute of Technology

Cambridge, MA 02139

tian@math.mit.edu

\section{V.S. Varadarajan}

University of California

Los Angeles, CA 90095-1555

vsv@math.ucla.edu

Dan Voiculescu

University of California

Berkeley, CA 94720

dvv@math.berkeley.edu

\section{SUPPORTING INSTITUTIONS}

CALIF. INST. OF TECHNOLOGY CHINESE UNIV. OF HONG KONG MACQUARIE UNIVERSITY NEW MEXICO STATE UNIV. OREGON STATE UNIV. PEKING UNIVERSITY RITSUMEIKAN UNIVERSITY STANFORD UNIVERSITY TOKYO INSTITUTE OF TECHNOLOGY UNIVERSIDAD DE LOS ANDES
UNIV. OF ARIZONA

UNIV. OF BRITISH COLUMBIA

UNIV. OF CALIF., BERKELEY

UNIV. OF CALIF., DAVIS

UNIV. OF CALIF,, IRVINE UNIV. OF CALIF., LOS ANGELES

UNIV. OF CALIF., RIVERSIDE UNIV. OF CALIF., SAN DIEGO UNIV. OF CALIF., SANTA BARBARA UNIV. OF CALIF., SANTA CRUZ
UNIV. OF HAWAII

UNIV. OF MELBOURNE

UNIV. OF MONTANA

UNIV. NACIONAL AUTONOMA DE MEXICO

UNIV. OF NEVADA, RENO

UNIV. OF OREGON

UNIV. OF SOUTHERN CALIFORNIA

UNIV OF UTAH

UNIV. OF WASHINGTON

WASHINGTON STATE UNIVERSITY

The supporting Institutions listed above contribute to the cost of publication of this Journal, but they are not owners or publishers and have no responsibility for its contents or policies.

Manuscripts must be prepared in accordance with the instructions provided on the inside back cover.

The table of contents and the abstracts of the papers in the current issue, as well as other information about the Pacific Journal of Mathematics, may be found on the Internet at http://www.math.uci.edu/pjm.html.

The Pacific Journal of Mathematics (ISSN 0030-8730) is published monthly except for July and August. Regular subscription rate: $\$ 245.00$ a year (10 issues). Special rate: $\$ 123.00$ a year to individual members of supporting institutions.

Subscriptions, back issues published within the last three years and changes of subscribers address should be sent to Pacific Journal of Mathematics, P.O. Box 4163, Berkeley, CA 94704-0163, U.S.A. Prior back issues are obtainable from Kraus Periodicals Co., Route 100, Millwood, NY 10546.

The Pacific Journal of Mathematics at the University of California, c/o Department of Mathematics, 981 Evans Hall, Berkeley, CA 94720 (ISSN 0030-8730) is published monthly except for July and August. Second-class postage paid at Berkeley, CA 94704, and additional mailing offices. POSTMASTER: send address changes to Pacific Journal of Mathematics, P.O. Box 6143, Berkeley, CA 94704-0163.

PUBLISHED BY PACIFIC JOURNAL OF MATHEMATICS at University of California, Berkeley, CA 94720, A NON-PROFIT CORPORATION

This publication was typeset using AMS-LATEX,

the American Mathematical Society's TEX macro system.

Copyright (c) 1995 by Pacific Journal of Mathematics 


\title{
PACIFIC JOURNAL OF MATHEMATICS
}

\author{
Volume $175 \quad$ No. $1 \quad$ September 1996
}

Homogeneous Ricci positive 5-manifolds

Dimitri Alekseevsky, Isabel Dotti de Miatello and CARlos J.

FERRARIS

On the structure of tensor products of $\ell_{p}$-spaces

ALVARO ARIAS and JEFFREY D. FARMER

The closed geodesic problem for compact Riemannian 2-orbifolds

JosEPH E. BORZELLINO and BENJAMIN G. LORICA

Small eigenvalue variation and real rank zero

Ola Bratteli and George A. Elliott

Global analytic hypoellipticity of $\square_{b}$ on circular domains

So-CHIN CHEN

Sharing values and a problem due to C. C. Yang

XIN-Hou HuA

Commutators and invariant domains for Schrödinger propagators

Min-JEI HUANG

Chaos of continuum-wise expansive homeomorphisms and dynamical properties of sensitive maps of graphs

\section{HiS AO KATO}

Some properties of Fano manifolds that are zeros of sections in homogeneous vector bundles over Grassmannians

\section{OLIVER KÜCHLE}

On polynomials orthogonal with respect to Sobolev inner product on the unit circle

XIN Li and FRANCISCO MARCELLAN

Maximal subfields of $\mathbf{Q}(i)$-division rings

STEVEN LIEDAHL

Virtual diagonals and $n$-amenability for Banach algebras

AlAN L. T. PATERSON

Rational Pontryagin classes, local representations, and $K^{G}$-theory

Claude Schochet

An equivalence relation for codimension one foliations of 3-manifolds

SANDRA SHIELDS

A construction of Lomonosov functions and applications to the invariant subspace problem

\section{ALEKSANDER SIMONIČ}

Complete intersection subvarieties of general hypersurfaces 